\title{
Controllability of fractional impulsive neutral stochastic functional differential equations via Kuratowski measure of noncompactness
}

\author{
Junhao $\mathrm{Hu}^{\mathrm{a}, *}$, Jiashun Yang ${ }^{\mathrm{a}}$, Chenggui Yuan ${ }^{\mathrm{b}}$ \\ a School of Mathematics and Statistics, South-Central University for Nationalities, Wuhan, 430074, China. \\ ${ }^{b}$ Department of Mathematics, Swansea University, Singleton Park, SA2 8PP, UK.
}

Communicated by X. Z. Liu

\begin{abstract}
In this paper, the controllability problem for a class of fractional impulsive neutral stochastic functional differential equations is considered in infinite dimensional space. By using Kuratowski measure of noncompactness and Mönch fixed point theorem, the sufficient conditions of controllability of the equations are obtained under the assumption that the semigroup generated by the linear part of the equations is not compact. At the end, an example is provided to illustrate the proposed result. (C)2017 All rights reserved.
\end{abstract}

Keywords: Controllability, fractional differential equations, impulsive stochastic differential equations, Kuratowski measure of noncompactness, Mönch fixed point theorem.

2010 MSC: 26A33, 65C30, 93B05.

\section{Introduction}

In recent years, various models of fractional differential equations in the fields of physics, chemistry, engineering, biology and economics have been proposed and examined. As a consequence, the theory of fractional differential equations has been studied by many authors, see $[13,18,21]$ and the references therein. A survey on existence results for boundary value problems of nonlinear fractional differential equations and inclusions was covered in Agarwal et al. [1]. The existence of mild solutions of a class of fractional neutral evolution equations was treated in Zhou and Jiao [32], and the controllability of a class of fractional-order neutral evolution control systems could be found in Sakthivel et al. [24].

On the other hand, many real models often fluctuate due to external stochastic perturbations [17]. The existence and uniqueness of solutions of neutral stochastic functional differential equations with infinite delay was discussed in Bao and Cao [4]. The p-th moment exponential stability and almost exponential stability of stochastic functional differential equations by using stochastic Razumikhin theorems were obtained in Mao [16]. The stability of neural stochastic functional differential equations was further extended in Huang and Deng [11]. The controllability conditions of semilinear stochastic delay evolution

\footnotetext{
*Corresponding author

Email addresses: junhaohu74@163.com (Junhao Hu), C.Yuan@swansea.ac.uk (Chenggui Yuan)
} 
equations in Hilbert spaces were established in Balasubramaniam and Dauer [2], and the approximate controllability of neutral stochastic functional differential systems with infinite delay was proved by Balasubramaniam et al. [3]. To state dependent delay, the existence of solution and approximate controllability of a second-order neutral stochastic differential equation were investigated by Das et al. [5]. Recently, the controllability of fractional stochastic differential systems have received a lot of attention. The controllability conditions of fractional neutral stochastic functional differential systems were derived in $\mathrm{Li}$ and Peng [15]. Approximate controllability of fractional neutral stochastic system with infinite delay and fractional stochastic evolution equations were found in [23, 25].

Besides stochastic perturbations, the impulsive effects may happen in the evolution systems which change abruptly at certain moments of time [14, 29]. The impulsive stabilization of impulsive delay differential system via the Lyapunov-Razumikhin method was studied in Wang and Liu [28]. The exponential stability and instability of impulsive stochastic functional differential equations with Markovian switching were given in Kao et al. [12]. The existence and controllability of fractional-order impulsive stochastic system with infinite delay were considered in [8]. The approximate controllability of fractional impulsive neutral stochastic differential equations with nonlocal conditions was explored in [31].

However, to the best of the authors' knowledge, the assumption of compactness of the semigroup generated by the linear part and the invertibility of the controllability operator of fractional impulsive stochastic evolution systems was imposed [8,31]. It turns out that in practice it is rather difficult to verify these conditions directly, and it fails in infinite dimensional space when the semigroup is compact, see $[26,27]$. Therefore, the exact controllability of nonlinear stochastic impulsive evolution differential inclustions with infinite delay in Hilbert spaces by using Kuratowski measure of noncompactness was investigated in Duan et al. [7]. Inspired by the previous work [7], in this paper, the controllability problem for a class of fractional impulsive neutral stochastic functional differential equations is considered in infinite dimensional space. The rest of the paper is organized as follows. In Section 2, some preliminaries such as some basic definitions, notation and lemmas are given. In Section 3, the sufficient conditions of controllability of the equations by using Kuratowski measure of noncompactness and Mönch fixed point theorem are obtained. Finally, in Section 4, an example is provided to illustrate the proposed result.

\section{Preliminaries}

In this section, we introduce definitions, notation, preliminary results needed to establish our main results. Let $(\mathrm{U},\langle\cdot, \cdot\rangle)$ and $(\mathrm{H},\langle\cdot, \cdot\rangle)$ denote two real separable Hilbert spaces, and $\mathcal{L}(\mathrm{U}, \mathrm{H})$ be the set of all linear bounded operators for $\mathrm{U}$ to $\mathrm{H}$. For convenience, we will use the same notation $\|\cdot\|$ to denote the norms in $\mathrm{U}, \mathrm{H}$ and $\mathcal{L}(\mathrm{U}, \mathrm{H})$. We assume that $\left(\mathcal{L}_{\mathrm{HS}}(\mathrm{H}),\|\cdot\|_{\mathrm{HS}}\right)$ is the space of all Hilbert-Schmidt operators from $\mathrm{H}$ to $\mathrm{H}$. Let $\left(\Omega, \mathcal{F},\left\{\mathcal{F}_{\mathfrak{t}}\right\}_{\mathfrak{t} \geqslant 0}, \mathbb{P}\right)$ be a complete probability space with a filtration $\left\{\mathcal{F}_{\mathfrak{t}}\right\}_{\mathrm{t} \geqslant 0}$ satisfying the usual conditions (i.e., it is increasing and right continuous, while $\mathcal{F}_{0}$ contains all $\mathbb{P}$-null sets). Suppose that $W(t)_{t \geqslant 0}$ is a cylindrical $H$-valued Wiener process with finite trace nuclear covariance operator $Q \geqslant 0$ defined on $\left(\Omega, \mathcal{F},\left\{\mathcal{F}_{t}\right\}_{t} \geqslant 0, \mathbb{P}\right)$. We consider the space $\mathcal{D}([-\tau, 0] ; H)=\{\zeta:[-\tau, 0] \rightarrow H: \zeta(t)$ is continuous except finite time $t=t_{i}, \zeta\left(t_{i}^{+}\right)$and $\zeta\left(t_{i}^{-}\right)$exist, and $\left.\zeta\left(t_{i}\right)=\zeta\left(t_{i}^{-}\right)\right\}$with the norm $\|\zeta\|=\sup _{-\tau \leqslant \theta \leqslant 0}\|\zeta(\theta)\|$.

In this paper, we are concerned with the controllability of the following fractional impulsive neutral stochastic functional differential equation

$$
\left\{\begin{array}{l}
{ }^{c} D_{t}^{\alpha}\left[x(t)-\lambda\left(t, x_{t}\right)\right]=A x(t)+B u(t)+f\left(t, x_{t}\right)+g\left(t, x_{t}\right) \frac{d W(t)}{d t}, \quad t \in J:=[0, b], \\
\left.\Delta x\right|_{t=t_{i}}=I_{i}\left(x\left(t_{i}^{-}\right)\right), \quad i=1,2, \cdots, \bar{s}, \\
x_{0}=\xi(t) \in \mathcal{D}([-\tau, 0], H),
\end{array}\right.
$$

where ${ }^{c} D_{t}^{\alpha}$ is the Caputo fractional derivative of order $\frac{1}{2}<\alpha<1$. $A: D(A) \subset H \rightarrow H$ is the infinitesimal generator of a strongly continuous semigroup of a bounded linear operator $\{T(t), t \geqslant 0\}$ in the Hilbert space $\mathrm{H}$. The control function $\mathrm{u}(\cdot)$ takes values in $\mathrm{L}^{2}(\mathrm{~J}, \mathrm{U})$ of admissible control functions for a separable Hilbert space $U, B$ is a bounded linear operator from $U$ into $H . f, \lambda: J \times \mathcal{D}([-\tau, 0] ; H) \rightarrow H$ and $g$ : 
$\mathrm{J} \times \mathcal{D}([-\tau, 0] ; \mathrm{H}) \rightarrow \mathcal{L}_{\mathrm{HS}}(\mathrm{H})$ are Borel measurable functions. $0<\mathrm{t}_{1}<\mathrm{t}_{2}<\cdots<\mathrm{t}_{\overline{\mathrm{s}}}<\mathrm{t}_{\overline{\mathrm{s}}+1}=\mathrm{b}$ are impulsive times, $\left.\Delta x\right|_{t=t_{i}}=x\left(t_{i}^{+}\right)-x\left(t_{i}^{-}\right), x\left(t_{i}^{+}\right)$and $x\left(t_{i}^{-}\right)$represent the right and left limits of $x(t)$ at $\mathrm{t}=\mathrm{t}_{\mathrm{i}}$, respectively. $\mathcal{P} \mathcal{C}([-\tau, b] ; H)=\left\{x:[-\tau, b] \rightarrow H: x(t)\right.$ is continuous except finite time $t=t_{i}$, $x\left(t_{i}^{+}\right)$and $x\left(t_{i}^{-}\right)$exist, and $\left.x\left(t_{i}\right)=x\left(t_{i}^{-}\right), i=1,2, \cdots, \bar{s}\right\}$, with the norm $\|x\|=\sup _{-\tau \leqslant \theta \leqslant b}\|x(\theta)\|$. In the following, $\mathcal{P C}:=\mathcal{P C}([-\tau, b] ; H)$, and $J_{0}:=\left[0, t_{1}\right], J_{i}:=\left(t_{i}, t_{i+1}\right], \cdots, J_{\bar{s}}:=\left(t_{\bar{s}}, b\right]$.

Definition 2.1. The fractional integral of order $\alpha$ of a function $h$ with the lower limit 0 is defined by

$$
I^{\alpha} h(t)=\frac{1}{\Gamma(\alpha)} \int_{0}^{t} \frac{h(s)}{(t-s)^{1-\alpha}} d s, \quad t>0, \quad \alpha>0,
$$

where $\Gamma(\cdot)$ is the gamma function.

Definition 2.2. The Caputo derivative of order $\alpha$ of a function $h$ with the lower limit 0 is defined by

$$
{ }^{c} D_{t}^{\alpha} h(t)=\frac{1}{\Gamma(n-\alpha)} \int_{0}^{t} \frac{h^{n}(s)}{(t-s)^{\alpha+1-n}} d s=I^{n-\alpha} h^{n}(t), \quad t>0, \quad n-1<\alpha<n .
$$

Definition 2.3. An $\mathcal{F}_{t}$-adapted stochastic process $\{x(t): t \in J\}$ is said to be a mild solution of system (2.1), if for all $\mathrm{t} \in[0, \mathrm{~b}], \mathbb{P}\left\{w: \int_{0}^{\mathrm{t}}\|x(\mathrm{~s})\|^{2} \mathrm{~d} s<\infty\right\}=1$, and

$$
\begin{aligned}
x(t)= & \eta(t) \xi(0)+\lambda\left(t, x_{t}\right)+\int_{0}^{t}(t-s)^{\alpha-1} \beta(t-s) B u(s) d s \\
& +\int_{0}^{t}(t-s)^{\alpha-1} \beta(t-s) f\left(s, x_{s}\right) d s \\
& +\int_{0}^{t}(t-s)^{\alpha-1} \beta(t-s) g\left(s, x_{s}\right) d W(s) \\
& +\sum_{0<t_{i}<t} \eta\left(t-t_{i}\right) I_{i}\left(x\left(t_{i}^{-}\right)\right), \text {a.s. } t \in J
\end{aligned}
$$

where

$$
\begin{array}{ll}
\eta(t)=\int_{0}^{\infty} \rho(\theta) \mathrm{T}\left(\mathrm{t}^{\alpha} \theta\right) \mathrm{d} \theta, & \beta(\mathrm{t})=\alpha \int_{0}^{\infty} \theta \rho(\theta) \mathrm{T}\left(\mathrm{t}^{\alpha} \theta\right) \mathrm{d} \theta, \\
\rho(\theta)=\frac{1}{\alpha} \theta^{-1-\frac{1}{\alpha}} \varpi\left(\theta^{-\frac{1}{\alpha}}\right) \geqslant 0, & \varpi(\theta)=\frac{1}{\pi} \sum_{n=1}^{\infty}(-1)^{n-1} \theta^{-n \alpha-1} \frac{\Gamma(\mathrm{n} \alpha+1)}{n !} \sin (\mathrm{n} \pi \alpha),
\end{array}
$$

and $\rho$ is a probability density function defined on $(0, \infty)$.

Definition 2.4. The system (2.1) is said to be controllable on J, if for every initial value $\xi(t)$, there exists a stochastic control $u \in L^{2}(J, U)$ such that the solution $x(\cdot)$ of (2.1) satisfies $x(b)=\bar{x}$, where $\bar{x} \in H$ is preassigned terminal state.

Lemma 2.5 ([32]). The operators $\eta, \beta$ have the following properties:

(i) For any $t \geqslant 0$, the operators $\eta(t), \beta(t)$ are linear and bounded, that is, there exists $L>0$, for any $x \in \mathrm{H}$,

$$
\|\eta(t) x\| \leqslant L\|x\|, \quad\|\beta(t) x\| \leqslant \frac{\alpha L}{\Gamma(1+\alpha)}\|x\| .
$$

(ii) The operators $\eta(t), \beta(t)$ are strongly continuous, that is, for any $x \in H, 0 \leqslant t^{\prime} \leqslant t^{\prime \prime}$, and $t^{\prime} \rightarrow t^{\prime \prime}$

$$
\left\|\eta\left(t^{\prime \prime}\right) x-\eta\left(t^{\prime}\right) x\right\| \rightarrow 0, \quad\left\|\beta\left(t^{\prime \prime}\right) x-\beta\left(t^{\prime}\right) x\right\| \rightarrow 0 .
$$


Remark 2.6. In Zang and $\mathrm{Li}[31]$, if $\mathrm{T}(\mathrm{t})$ is compact for every $t>0, \eta(t), \beta(t)$ are also compact operators. However, in this paper, the compactness of the operators $\eta$ and $\beta$ are not considered for controllability of system (2.1).

Definition 2.7. Let $(\mathcal{A}, \rho)$ be a partially ordered set. $\mathrm{D}$ is a bounded subset of $\mathrm{H}$. A function $\mu: \mathrm{H} \rightarrow \mathcal{A}$ is called a Kuratowski measure of noncompactness in $\mathrm{H}$, if

$$
\mu(D)=\inf \left\{\epsilon>0: D \subset \bigcup_{i=1}^{m} D_{i}, \operatorname{diam}\left(D_{i}\right) \leqslant \epsilon\right\} .
$$

Lemma 2.8 ([10]). Let $V=\left\{f_{n}\right\} \subset L^{1}(J, H)$. If there exists $h \in L^{1}\left(J, \mathbb{R}^{+}\right)$, such that for $t \in J,\left\|f_{n}(t)\right\| \leqslant h(t)$, then

$$
\mu(V(t)) \in \mathrm{L}^{1}\left(J, \mathbb{R}^{+}\right), \quad \mu\left(\left\{\int_{0}^{t} f_{n}(s) d s: n \in \mathbb{N}\right\}\right) \leqslant 2 \int_{0}^{t} \mu(V(s)) d s, \quad t \in J,
$$

where $\mathrm{V}(\mathrm{t})=\{\mathrm{x}(\mathrm{t}): \mathrm{x} \in \mathrm{V}\} \subseteq \mathrm{H}$.

Lemma 2.9 ([30]). If $V \subset \mathcal{P C}$ is bounded and piecewise equicontinuous, then $\mu(V(t))$ is also piecewise equicontinuous on $\mathrm{J}$, and

$$
\mu(V)=\sup \{\mu(V(t)): t \in J\}
$$

where $\mathrm{V}(\mathrm{t})=\{x(\mathrm{t}): \mathrm{x} \in \mathrm{V}\} \subseteq \mathrm{H}$.

Lemma 2.10 ([9]). If $V \subset \mathcal{P C}$ is bounded, and equicontinuous on $\mathrm{J}_{i}, 0=1,2, \cdots, \overline{\mathrm{s}}$, then

$$
\mu(V)=\max _{0 \leqslant i \leqslant s}\left(V\left(J_{i}\right)\right)=\max _{0 \leqslant i \leqslant s} \sup \left\{\mu(V(t)): t \in J_{i}\right\}
$$

Lemma 2.11 ([19]). Let $D$ be a bounded closed and convex subset of $H, 0 \in D$. F : D $\rightarrow H$ is continuous, such that for any countable set $M \subseteq D, M \subseteq \overline{\operatorname{conv}}(\{0\} \cup F(M)), M$ is relatively compact. Then $F$ has a fixed point in D.

\section{Main results}

To get the controllability, we need the following assumptions.

(H1) The function $f$ satisfies that

(1) for any $x \in \mathcal{D}([-\tau, 0] ; H)$, the function $t \mapsto f(t, x)$ is strong measurable, for any $t \in J_{i}$, the function $x \mapsto f(t, x)$ is continuous;

(2) there exist a bounded function $p(t): J \rightarrow \mathbb{R}^{+}$, and a continuous nondecreasing function $\varphi$ : $[0, \infty) \rightarrow[0, \infty)$ such that

$$
\|f(t, x)\|^{2} \leqslant p(t) \varphi\left(\|x\|^{2}\right), \quad \liminf _{n \rightarrow \infty} \frac{\varphi(n)}{n}=0 ;
$$

(3) there exists $\mathrm{K}_{\mathrm{f}} \in \mathrm{L}^{1}\left(\mathrm{~J} ; \mathbb{R}^{+}\right)$, for any bounded set $\mathrm{D} \subset \mathcal{P C}$,

$$
\mu\left(f\left(t, D_{t}\right)\right) \leqslant K_{f}(t) \mu\left(D_{t}\right),
$$

where $D_{t}:=\left\{x_{t}: x \in D\right\} \subseteq \mathcal{D}([-\tau, 0] ; H)$.

(H2) The function $g$ satisfies that

(1) for any $x \in \mathcal{D}([-\tau, 0] ; H)$, the function $t \mapsto g(t, x)$ is strong measurable, for any $t \in J_{i}$, the function $x \mapsto g(t, x)$ is continuous;

(2) there exists $\psi_{\mathrm{n}} \in \mathrm{L}^{1}\left(\mathrm{~J} ; \mathbb{R}^{+}\right)$, such that

$$
\sup _{\|x\| \leqslant n}\|g(t, x)\|^{2} \leqslant \psi_{n}(t) \text {, a.e., }
$$

and

$$
\liminf _{n \rightarrow \infty} \frac{\int_{0}^{b}(b-s)^{2 \alpha-2} \psi_{n}(s) d s}{n}=0 ;
$$


(3) there exists $\mathrm{K}_{\mathrm{g}} \in \mathrm{L}^{1}\left(\mathrm{~J} ; \mathbb{R}^{+}\right)$, for any bounded set $\mathrm{D} \subset \mathcal{P C}$,

$$
\mu\left(g\left(t, D_{t}\right)\right) \leqslant K_{g}(t) \mu\left(D_{t}\right),
$$

where $\mathrm{D}_{\mathrm{t}}:=\left\{x_{\mathrm{t}}: x \in \mathrm{D}\right\} \subseteq \mathcal{D}([-\tau, 0] ; \mathrm{H})$.

(H3) The neutral function $\lambda$ satisfies that

(1) for any $x \in \mathcal{D}([-\tau, 0] ; H)$, the function $t \mapsto \lambda(t, x)$ is piecewise continuous, for any $t \in J_{i}$, the function $x \mapsto \lambda(t, x)$ is continuous;

(2) there exist a bounded function $\mathrm{q}(\mathrm{t}): \mathrm{J} \rightarrow \mathbb{R}^{+}$, and a continuous nondecreasing $\phi:[0, \infty) \rightarrow$ $[0, \infty)$ such that

$$
\|\lambda(t, x)\|^{2} \leqslant q(t) \phi\left(\|x\|^{2}\right), \quad \liminf _{n \rightarrow \infty} \frac{\phi(n)}{n}=0 ;
$$

(3) there exists $K_{\lambda} \in L^{1}\left(J ; \mathbb{R}^{+}\right)$, for any bounded $D \subset \mathcal{P C}$,

$$
\mu\left(\lambda\left(t, D_{t}\right)\right) \leqslant K_{\lambda}(t) \mu\left(D_{t}\right)
$$

where $\mathrm{D}_{\mathrm{t}}:=\left\{x_{\mathrm{t}}: x \in \mathrm{D}\right\} \subseteq \mathcal{D}([-\tau, 0] ; \mathrm{H})$.

(H4) The impulsive function $\mathrm{I}_{i}: \mathrm{H} \rightarrow \mathrm{H}, \mathrm{i}=1,2, \cdots, \overline{\mathrm{s}}$ is continuous such that

(1) there exist nondecreasing functions $\mathrm{L}_{i}: \mathbb{R}^{+} \rightarrow \mathbb{R}^{+}$,

$$
\left\|I_{i}(x)\right\|^{2} \leqslant L_{i}\left(\|x\|^{2}\right), \quad x \in H, \quad \liminf _{n \rightarrow \infty} \frac{L_{i}(n)}{n}=0, \quad i=1,2, \cdots, \bar{s} ;
$$

(2) there exist $K_{i} \geqslant 0$, for any bounded set $D \subset \mathcal{P C}$,

$$
\mu\left(I_{i}(D)\right) \leqslant K_{i} \mu(D), \quad i=1,2, \cdots, \bar{s} .
$$

(H5) $\mathrm{B}: \mathrm{U} \rightarrow \mathrm{H}$ is a bounded linear operator, $\Theta: \mathrm{L}^{2}(\mathrm{~J}, \mathrm{U}) \rightarrow \mathrm{H}$ is linear operator defined by

$$
\Theta u=\int_{0}^{b}(b-s)^{\alpha-1} \beta(b-s) B u(s) d s,
$$

and

(1) the operator $\Theta$ has an invertible operator $\Theta^{-1}$ which takes values in $\mathrm{L}^{2}(\mathrm{~J}, \mathrm{U}) \backslash \operatorname{Ker} \Theta$ (see [22, 26]), and there exist positive constants $L_{B}$ and $L_{\Theta}$, such that

$$
\|\mathrm{B}\| \leqslant \mathrm{L}_{\mathrm{B}}, \quad\left\|\Theta^{-1}\right\| \leqslant \mathrm{L}_{\Theta}
$$

(2) there exist $\mathrm{K}_{\Theta}(t) \in \mathrm{L}^{1}\left(\mathrm{~J}, \mathbb{R}^{+}\right), \mathrm{K}_{\mathrm{B}} \geqslant 0$, for any bounded sets $\mathrm{D}_{1} \subset \mathrm{H}, \mathrm{D}_{2} \subset \mathrm{U}$,

$$
\mu\left(\left(\Theta^{-1} D_{1}\right)(t)\right) \leqslant K_{\Theta}(t) \mu\left(D_{1}(t)\right), \quad \mu\left(B\left(D_{2}\right)\right) \leqslant K_{B} \mu_{u}\left(D_{2}\right) .
$$

(H6) Assume the following inequality holds:

$$
\begin{aligned}
\bar{l}= & \left(1+\frac{\mathrm{LK}_{\mathrm{B}}\left\|\mathrm{K}_{\Theta}\right\|_{\mathrm{L}^{1}} \mathrm{~b}^{\alpha}}{\Gamma(1+\alpha)}\right) \times\left(\frac{2 \mathrm{Lb}^{\alpha}}{\Gamma(1+\alpha)}\left\|\mathrm{K}_{\mathrm{f}}\right\|_{\mathrm{L}^{1}}+\sum_{i=1}^{\bar{s}} \mathrm{LK}_{i}+\left\|\mathrm{K}_{\lambda}\right\|_{\mathrm{L}^{1}}\right. \\
& \left.+\frac{\alpha \mathrm{Lb}^{\alpha-1 / 2}}{\sqrt{(2 \alpha-1)} \Gamma(1+\alpha)}\left\|\mathrm{K}_{\mathrm{g}}\right\|_{\mathrm{L}^{1}}\right)<1 .
\end{aligned}
$$

Theorem 3.1. Assume that the conditions (H1)-(H6) are satisfied, then the system (2.1) is controllable on $\mathrm{J}$. 
Proof. By assumption (H2) (1), for any $x \in \mathcal{P C}$, we define the control

$$
\begin{aligned}
\bar{u}_{x}(t)= & \Theta^{-1}\left[\bar{x}-\eta(b) \xi(0)-\lambda\left(b, \bar{x}_{b}\right)-\int_{0}^{b}(b-s)^{\alpha-1} \beta(b-s) f\left(s, x_{s}\right) d s\right. \\
& \left.-\int_{0}^{b}(b-s)^{\alpha-1} \beta(b-s) g\left(s, x_{s}\right) d W(s)-\sum_{i=1}^{\bar{s}} \eta\left(b-t_{i}\right) I_{i}\left(x\left(t_{i}^{-}\right)\right)\right](t) .
\end{aligned}
$$

And using the control function $\bar{u}_{x}(t)$, define the operator $F_{1}$ :

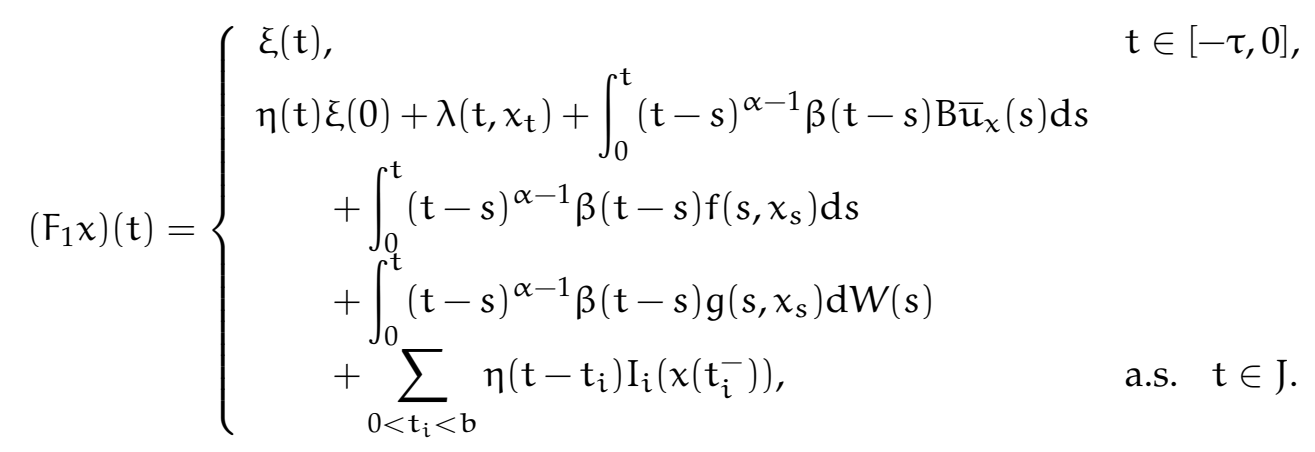

We shall show that $F_{1}$ has a fixed point, which is a mild solution of system $(2.1)$. Clearly, $x(b)=\left(F_{1} x\right)(b)=$ $\bar{\chi}$, that is, the system (2.1) is controllable on $\mathrm{J}$.

For any $\xi \in \mathcal{D}([-\tau, 0], H)$, define

$$
\widehat{\xi}(t)= \begin{cases}\xi(t), & t \in[-\tau, 0], \\ \eta(t) \xi(0), & \text { a.s. } t \in J .\end{cases}
$$

Set $x(t)=y(t)+\widehat{\xi}(t)$. Let $\mathcal{P} \mathcal{C}_{0}=\left\{y \in \mathcal{P C}: y_{0}=0\right\}$. Consider $G_{1}: \mathcal{P C}_{0} \rightarrow \mathcal{P C}_{0}$ defined by

$$
\left(G_{1} y\right)(t)=\left\{\begin{array}{ccc}
0, & t \in[-\tau, 0], \\
\lambda\left(t, y_{t}+\widehat{\xi}_{t}\right)+\int_{0}^{t}(t-s)^{\alpha-1} \beta(t-s) B \bar{u}_{y}(s) d s & \\
& +\int_{0}^{t}(t-s)^{\alpha-1} \beta(t-s) f\left(s, y_{s}+\widehat{\xi}_{s}\right) d s & \\
& +\int_{0}^{t}(t-s)^{\alpha-1} \beta(t-s) g\left(s, y_{s}+\widehat{\xi}_{s}\right) d W(s) & \\
& +\sum_{0<t_{i}<t} \eta\left(t-t_{i}\right) I_{i}\left(y\left(t_{i}^{-}\right)+\widehat{\xi}\left(t_{i}^{-}\right)\right), & \text {a.s. } t \in J,
\end{array}\right.
$$

where

$$
\begin{aligned}
\bar{u}_{y}(t)= & \Theta^{-1}\left[\bar{x}-\eta(b) \xi(0)-\lambda\left(b, y_{b}+\widehat{\xi}_{b}\right)-\int_{0}^{b}(b-s)^{\alpha-1} \beta(b-s) f\left(s, y_{s}+\widehat{\xi}_{s}\right) d s\right. \\
& \left.-\int_{0}^{b}(b-s)^{\alpha-1} \beta(b-s) g\left(s, y_{s}+\widehat{\xi}_{s}\right) d W(s)-\sum_{i=1}^{\bar{s}} \eta\left(b-t_{i}\right) I_{i}\left(y\left(t_{i}^{-}\right)+\widehat{\xi}\left(t_{i}^{-}\right)\right)\right](t) .
\end{aligned}
$$

Then, it is clear that $F_{1}$ has a fixed point if and only if $G_{1}$ has a fixed point. Therefore, we divide the proof into four steps.

Step 1. By contradiction, we will show that there exists $n_{0} \geqslant 1$, such that $G_{1}\left(B_{n_{0}}\right) \subseteq B_{n_{0}}$, where $B_{n_{0}}=$ $\left\{y \in \mathcal{P C}_{0},\|y\| \leqslant n_{0}\right\}$.

If it is not true, for any $n \geqslant 1$, there exists $y^{*}(\cdot) \in B_{n}$, such that $G_{1} y^{*} \notin B_{n}$. 
In fact, we have from (H1)-(H4) that

$$
\begin{aligned}
n^{2} \leqslant & \mathbb{E}\left\|G_{1} y^{*}(t)\right\|^{2} \leqslant(\bar{s}+4) \mathbb{E}\left\|\lambda\left(t, y_{t}^{*}+\widehat{\xi}_{t}\right)\right\|^{2} \\
& +(\bar{s}+4) \mathbb{E}\left\|\int_{0}^{b}(b-s)^{\alpha-1} \beta(b-s) B \bar{u}_{y^{*}}(s) d s\right\|^{2} \\
& +(\bar{s}+4) \mathbb{E}\left\|\int_{0}^{b}(b-s)^{\alpha-1} \beta(b-s) f\left(s, y_{s}^{*}+\widehat{\xi}_{s}\right) d s\right\|^{2} \\
& +(\bar{s}+4) \mathbb{E}\left\|\int_{0}^{b}(b-s)^{\alpha-1} \beta(b-s) g\left(s, y_{s}^{*}+\widehat{\xi}_{s}\right) d W(s)\right\|^{2} \\
& +(\bar{s}+4) \sum_{i=1}^{\bar{s}}\left\|\eta\left(b-t_{i}\right) I_{i}\left(y^{*}\left(t_{i}^{-}\right)+\widehat{\xi}\left(t_{i}^{-}\right)\right)\right\|^{2} .
\end{aligned}
$$

By Hölder's inequality, and Lemma 2.5, we have

$$
\begin{aligned}
\mathbb{E}\left\|\overline{\mathrm{u}}_{\mathrm{y}^{*}}\right\|^{2}= & (\overline{\mathrm{s}}+5) \mathrm{L}_{\Theta}^{2}\left[\|\overline{\mathrm{x}}\|^{2}+\mathrm{L}^{2}\|\xi\|^{2}+\sup _{\mathrm{t} \in \mathrm{J}} \mathrm{q}(\mathrm{t}) \phi\left(\mathrm{n}^{\prime 2}\right)\right. \\
& +\sup _{\mathrm{t} \in \mathrm{J}} \mathrm{p}(\mathrm{t}) \frac{\mathrm{b}^{2 \alpha+1}}{\alpha^{2}}\left(\frac{\alpha \mathrm{L}}{\Gamma(1+\alpha)}\right)^{2} \varphi\left(\mathrm{n}^{\prime 2}\right) \\
& +\left(\frac{\alpha \mathrm{L}}{\Gamma(1+\alpha)}\right)^{2} \int_{0}^{\mathrm{b}}(\mathrm{b}-\mathrm{s})^{2 \alpha-2} \psi_{\mathrm{n}^{\prime}}(\mathrm{s}) \mathrm{ds} \\
& \left.+\sum_{i=1}^{\bar{s}}\left\|\eta\left(\mathrm{b}-\mathrm{t}_{\mathrm{i}}\right) \mathrm{I}_{\mathrm{i}}\left(\mathrm{n}^{\prime}\right)\right\|^{2}\right]
\end{aligned}
$$

where $n^{\prime}=n+(L+1)\|\xi\|$.

Substituting (3.2) into (3.1) yields that

$$
\begin{aligned}
\mathrm{n}^{2} \leqslant & (\bar{s}+4)(\bar{s}+5) \mathrm{L}_{\Theta}^{2} \mathrm{~L}_{\mathrm{B}}^{2} \frac{\mathrm{b}^{2 \alpha+1}}{\alpha^{2}}\left(\frac{\alpha \mathrm{L}}{\Gamma(1+\alpha)}\right)^{2}\left[\|\overline{\mathrm{x}}\|^{2}+\mathrm{L}^{2}\|\xi\|^{2}\right] \\
& +\left[(\bar{s}+4)+(\overline{\mathrm{s}}+4)(\overline{\mathrm{s}}+5) \mathrm{L}_{\Theta}^{2} \mathrm{~L}_{\mathrm{B}}^{2} \frac{\mathrm{b}^{2 \alpha+1}}{\alpha^{2}}\left(\frac{\alpha \mathrm{L}}{\Gamma(1+\alpha)}\right)^{2}\right]\left[\sup _{\mathrm{t} \in \mathrm{J}} \mathrm{q}(\mathrm{t}) \phi\left(\mathrm{n}^{\prime 2}\right)+\sum_{\mathrm{i}=1}^{\bar{s}} \mathrm{~L}^{2} \mathrm{~L}_{\mathrm{i}}\left(\mathrm{n}^{\prime 2}\right)\right] \\
& +\left[(\overline{\mathrm{s}}+4)(\overline{\mathrm{s}}+5) \mathrm{L}_{\Theta}^{2} \mathrm{~L}_{\mathrm{B}}^{2} \frac{\mathrm{b}^{4 \alpha+2}}{\alpha^{4}}\left(\frac{\alpha \mathrm{L}}{\Gamma(1+\alpha)}\right)^{4}+(\overline{\mathrm{s}}+4) \frac{\mathrm{b}^{2 \alpha+1}}{\alpha^{2}}\left(\frac{\alpha \mathrm{L}}{\Gamma(1+\alpha)}\right)^{2}\right] \sup _{\mathrm{t} \in \mathrm{J}} p(\mathrm{t}) \varphi\left(\mathrm{n}^{\prime 2}\right) \\
& +\left[(\bar{s}+4)(\bar{s}+5) \mathrm{L}_{\Theta}^{2} \mathrm{~L}_{\mathrm{B}}^{2} \frac{\mathrm{b}^{2 \alpha+1}}{\alpha^{2}}\left(\frac{\alpha \mathrm{L}}{\Gamma(1+\alpha)}\right)^{4}+(\overline{\mathrm{s}}+4)\left(\frac{\alpha \mathrm{L}}{\Gamma(1+\alpha)}\right)^{2}\right] \int_{0}^{\mathrm{b}}(\mathrm{b}-\mathrm{s})^{2 \alpha-2} \psi_{\mathrm{n}^{\prime}}(\mathrm{s}) \mathrm{d} s
\end{aligned}
$$

Dividing both sides by $n^{2}$, we obtain from (3.3) that

$$
\begin{aligned}
1 \leqslant & (\bar{s}+4)(\bar{s}+5) \mathrm{L}_{\Theta}^{2} \mathrm{~L}_{\mathrm{B}}^{2} \frac{\mathrm{b}^{2 \alpha+1}}{\mathrm{n}^{2} \alpha^{2}}\left(\frac{\alpha \mathrm{L}}{\Gamma(1+\alpha)}\right)^{2}\left[\|\bar{x}\|^{2}+\mathrm{L}^{2}\|\xi\|^{2}\right] \\
& +\left[(\bar{s}+4)+(\bar{s}+4)(\bar{s}+5) \mathrm{L}_{\Theta}^{2} \mathrm{~L}_{\mathrm{B}}^{2} \frac{\mathrm{b}^{2 \alpha+1}}{\alpha^{2}}\left(\frac{\alpha \mathrm{L}}{\Gamma(1+\alpha)}\right)^{2}\right]\left[\sup _{\mathrm{t} \in \mathrm{J}} \mathrm{q}(\mathrm{t}) \frac{\phi\left(\mathrm{n}^{\prime 2}\right)}{\mathrm{n}^{2}}+\sum_{i=1}^{\bar{s}} \mathrm{~L}^{2} \frac{\mathrm{L}_{\mathrm{i}}\left(\mathrm{n}^{\prime 2}\right)}{\mathrm{n}^{2}}\right] \\
& +\left[(\overline{\mathrm{s}}+4)(\overline{\mathrm{s}}+5) \mathrm{L}_{\Theta}^{2} \mathrm{~L}_{\mathrm{B}}^{2} \frac{\mathrm{b}^{4 \alpha+2}}{\alpha^{4}}\left(\frac{\alpha \mathrm{L}}{\Gamma(1+\alpha)}\right)^{4}+(\bar{s}+4) \frac{\mathrm{b}^{2 \alpha+1}}{\alpha^{2}}\left(\frac{\alpha \mathrm{L}}{\Gamma(1+\alpha)}\right)^{2}\right] \sup _{\mathrm{t} \in \mathrm{J}} p(\mathrm{t}) \frac{\varphi\left(\mathrm{n}^{\prime 2}\right)}{\mathrm{n}^{2}} \\
& +\left[(\bar{s}+4)(\bar{s}+5) \mathrm{L}_{\Theta}^{2} \mathrm{~L}_{\mathrm{B}}^{2} \frac{\mathrm{b}^{2 \alpha+1}}{\alpha^{2}}\left(\frac{\alpha \mathrm{L}}{\Gamma(1+\alpha)}\right)^{4}+(\bar{s}+4)\left(\frac{\alpha \mathrm{L}}{\Gamma(1+\alpha)}\right)^{2}\right] \frac{1}{n^{2}} \int_{0}^{b}(b-s)^{2 \alpha-2} \psi_{n^{\prime}}(s) \mathrm{d} s .
\end{aligned}
$$


Taking the limit as $n \rightarrow \infty$, this contradicts with our assumptions. Thus, there exists $n_{0} \geqslant 1$, such that $\mathrm{G}_{1}\left(\mathrm{~B}_{\mathrm{n}_{0}}\right) \subseteq \mathrm{B}_{\mathrm{n}_{0}}$.

Step 2. we show that the operator $G_{1}: B_{n_{0}} \rightarrow B_{n_{0}}$ is continuous. Let $\left\{y^{(m)}(t)\right\}_{m=1}^{\infty} \subseteq B_{n_{0}}$, and $y^{(m)} \rightarrow$ $\mathrm{y} \in \mathrm{B}_{\mathrm{n}_{0}}$. By Hölder's inequality, we have

$$
\begin{aligned}
& \mathbb{E} \|\left(G_{1} y^{(m)}(t)-\left(G_{1} y\right)(t) \|^{2}\right. \\
& \leqslant(\bar{s}+4) \mathbb{E}\left\|\lambda\left(t, y_{t}^{(m)}+\widehat{\xi}_{t}\right)-\lambda\left(t, y_{t}+\widehat{\xi}_{t}\right)\right\|^{2} \\
&+(\bar{s}+4) \int_{0}^{t}\left|(t-s)^{\alpha-1} \beta(t-s)\right|^{2} d s \mathbb{E} \int_{0}^{t}\left\|B u_{y^{(m)}}(s)-B u_{y}(s)\right\|^{2} d s \\
&+(\bar{s}+4) \int_{0}^{t}\left|(t-s)^{\alpha-1} \beta(t-s)\right|^{2} d s \mathbb{E} \int_{0}^{t}\left\|f\left(s, y_{s}^{(m)}+\widehat{\xi}_{s}\right)-f\left(s, y_{s}+\widehat{\xi}_{s}\right)\right\|^{2} d s \\
&+(\bar{s}+4) \mathbb{E} \int_{0}^{t}\left\|(t-s)^{\alpha-1} \beta(t-s)\left(g\left(s, y_{s}^{(m)}+\widehat{\xi}_{s}\right)-g\left(s, y_{s}+\widehat{\xi}_{s}\right)\right)\right\|^{2} d s \\
&+(\bar{s}+4) \sum_{i=1}^{\bar{s}}\left\|\eta\left(t-t_{i}\right)\left(I_{i}\left(y(m)\left(t_{i}^{-}\right)+\widehat{\xi}_{s}\left(t_{i}^{-}\right)\right)-I_{i}\left(y\left(t_{i}^{-}\right)+\widehat{\xi}_{(t}\left(t_{i}^{-}\right)\right)\right)\right\|^{2} \\
& \leqslant(\bar{s}+4) \mathbb{E}\left\|\lambda\left(t, y_{t}^{(m)}+\widehat{\xi}_{t}\right)-\lambda\left(t, y_{t}+\widehat{\xi}_{t}\right)\right\|^{2} \\
&+\frac{(\bar{s}+4) L_{B}^{2} b^{\alpha} L^{2}}{(\Gamma(1+\alpha))^{2}} \mathbb{E} \int_{0}^{t}\left\|u_{y^{(m)}}(s)-u_{y}(s)\right\|^{2} d s \\
&+\frac{(\bar{s}+4) b^{\alpha} L^{2}}{(\Gamma(1+\alpha))^{2}} \mathbb{E} \int_{0}^{t}\left\|f\left(s, y_{s}^{(m)}+\widehat{\xi}_{s}\right)-f\left(s, y_{s}+\widehat{\xi}_{s}\right)\right\|^{2} d s \\
&+(\bar{s}+4) \mathbb{E} \int_{0}^{t}\left\|(t-s)^{\alpha-1} \beta(t-s)\left(g\left(s, y_{s}^{(m)}+\widehat{\xi}_{s}\right)-g\left(s, y_{s}+\widehat{\xi}_{s}, r(s)\right)\right)\right\|^{2} d s \\
&+(\bar{s}+4) L^{2} \sum_{i=1}^{s}\left\|\left(I_{i}\left(y^{(m)}\left(t_{i}^{-}\right)+\widehat{\xi}_{(t}\left(t_{i}^{-}\right)\right)-I_{i}\left(y\left(t_{i}^{-}\right)+\widehat{\xi}\left(t_{i}^{-}\right)\right)\right)\right\|^{2} .
\end{aligned}
$$

For the control function, by (H5), one can derive that

$$
\begin{aligned}
\mathbb{E}\left\|\bar{u}_{y^{(m)}}-\bar{u}_{y}\right\|^{2} \leqslant & (\bar{s}+3) \mathrm{L}_{\Theta}^{2} \mathbb{E}\left\|\lambda\left(b, y_{b}^{(m)}+\widehat{\xi}_{b}\right)-\lambda\left(b, y_{b}+\widehat{\xi}_{b}\right)\right\|^{2} \\
& +\frac{(\bar{s}+3) \mathrm{L}_{\Theta}^{2} b^{\alpha} \mathrm{L}^{2}}{(\Gamma(1+\alpha))^{2}} \mathbb{E} \int_{0}^{b}\left\|f\left(s, y_{s}^{(m)}+\widehat{\xi}_{s}\right)-f\left(s, y_{s}+\widehat{\xi}_{s}\right)\right\|^{2} d s \\
& +(\bar{s}+3) \mathrm{L}_{\Theta}^{2} \mathbb{E} \int_{0}^{b}\left\|(b-s)^{\alpha-1} \beta(b-s)\left(g\left(s, y_{s}^{(m)}+\widehat{\xi}_{s}\right)-g\left(s, y_{s}+\widehat{\xi}_{s}\right)\right)\right\|^{2} d s \\
& +(\bar{s}+3) L^{2} L_{\Theta}^{2} \sum_{i=1}^{\bar{s}}\left\|\left(I_{i}\left(y^{(m)}\left(t_{i}^{-}\right)+\widehat{\xi}\left(t_{i}^{-}\right)\right)-I_{i}\left(y\left(t_{i}^{-}\right)+\widehat{\xi}\left(t_{i}^{-}\right)\right)\right)\right\|^{2} .
\end{aligned}
$$

Substituting this into (3.4), by Lebesgue's dominated convergence theorem and (H1)-(H4), we see that $\mathbb{E}\left\|G_{1} y^{(m)}-G_{1} y\right\|^{2} \rightarrow 0$ as $y^{(m)} \rightarrow y$.

Step 3. The operator $G_{1}$ is equicontinuous on $J_{i}, i=1,2, \cdots, \bar{s}$. For any $t_{1}, t_{2} \in J_{i}$, and $t_{1}<t_{2}, y \in B_{n_{0}}$

$$
\begin{aligned}
\mathbb{E} \|\left(G_{1} y\right)\left(t_{2}\right)- & \left(G_{1} y\right)\left(t_{1}\right) \|^{2} \\
\leqslant & 11 \mathbb{E}\left\|\lambda\left(t_{2}, y_{t_{2}}+\widehat{\xi}_{t_{2}}\right)-\lambda\left(t_{1}, y_{t_{1}}+\widehat{\xi}_{t_{1}}\right)\right\|^{2} \\
& +11 \mathbb{E}\left\|\int_{0}^{t_{1}}\left(t_{1}-s\right)^{\alpha-1}\left[\beta\left(t_{2}-s\right)-\beta\left(t_{1}-s\right)\right] B \bar{u}_{y}(s) d s\right\|^{2}
\end{aligned}
$$




$$
\begin{aligned}
& +11 \mathbb{E}\left\|\int_{0}^{t_{1}}\left[\left(t_{2}-s\right)^{\alpha-1}-\left(t_{1}-s\right)^{\alpha-1}\right] \beta\left(t_{2}-s\right) B \bar{u}_{y}(s) d s\right\|^{2} \\
& +11 \mathbb{E}\left\|\int_{t_{1}}^{t_{2}}\left(t_{2}-s\right)^{\alpha-1} \beta\left(t_{2}-s\right) B \bar{u}_{y}(s) d s\right\|^{2} \\
& +11 \mathbb{E}\left\|\int_{0}^{t_{1}}\left(t_{1}-s\right)^{\alpha-1}\left[\beta\left(t_{2}-s\right)-\beta\left(t_{1}-s\right)\right] f\left(s, y_{s}+\widehat{\xi}_{s}\right) d s\right\|^{2} \\
& +11 \mathbb{E}\left\|\int_{0}^{t_{1}}\left[\left(t_{2}-s\right)^{\alpha-1}-\left(t_{1}-s\right)^{\alpha-1}\right] \beta\left(t_{2}-s\right) f\left(s, y_{s}+\widehat{\xi}_{s}\right) d s\right\|^{2} \\
& +11 \mathbb{E}\left\|\int_{t_{1}}^{t_{2}}\left(t_{2}-s\right)^{\alpha-1} \beta\left(t_{2}-s\right) f\left(s, y_{s}+\widehat{\xi}_{s}\right) d s\right\|^{2} \\
& +11 \mathbb{E}\left\|\int_{0}^{t_{1}}\left(t_{1}-s\right)^{\alpha-1}\left[\beta\left(t_{2}-s\right)-\beta\left(t_{1}-s\right)\right] g\left(s, y_{s}+\widehat{\xi}_{s}\right) d W(s)\right\|^{2} \\
& +11 \mathbb{E}\left\|\int_{0}^{t_{1}}\left[\left(t_{2}-s\right)^{\alpha-1}-\left(t_{1}-s\right)^{\alpha-1}\right] \beta\left(t_{2}-s\right) g\left(s, y_{s}+\widehat{\xi}_{s}\right) d W(s)\right\|^{2} \\
& +11 \mathbb{E}\left\|\int_{t_{1}}^{t_{2}}\left(t_{2}-s\right)^{\alpha-1} \beta\left(t_{2}-s\right) g\left(s, y_{s}+\widehat{\xi}_{s}\right) d W(s)\right\|^{2} \\
& +11 \mathbb{E}\left\|\sum_{i=1}^{s}\left(\eta\left(t_{2}-t_{i}\right)-\eta\left(t_{1}-t_{i}\right)\right) I_{i}\left(y\left(t_{i}^{-}\right)+\widehat{\xi}\left(t_{i}^{-}\right)\right)\right\|^{2} .
\end{aligned}
$$

By Hölder's inequality, we derive that

$$
\begin{aligned}
& \mathbb{E}\left\|\left(G_{1} y\right)\left(t_{2}\right)-\left(G_{1} y\right)\left(t_{1}\right)\right\|^{2} \\
& \leqslant 22 \mathbb{E}\left\|\lambda\left(t_{2}, y_{t_{2}}+\widehat{\xi}_{t_{2}}\right)-\lambda\left(t_{1}, y_{t_{2}}+\widehat{\xi}_{t_{2}}\right)\right\|^{2} \\
& +22 \mathbb{E}\left\|\lambda\left(t_{1}, y_{t_{2}}+\widehat{\xi}_{t_{2}}\right)-\lambda\left(t_{1}, y_{t_{1}}+\widehat{\xi}_{t_{1}}\right)\right\|^{2} \\
& +11 \int_{0}^{t_{1}}\left|\left(t_{1}-s\right)^{\alpha-1}\left[\beta\left(t_{2}-s\right)-\beta\left(t_{1}-s\right)\right]\right|^{2} d s \mathbb{E} \int_{0}^{b}\left\|B \bar{u}_{y}(s)\right\|^{2} d s \\
& +11 \int_{0}^{t_{1}}\left|\left[\left(t_{2}-s\right)^{\alpha-1}-\left(t_{1}-s\right)^{\alpha-1}\right] \beta\left(t_{2}-s\right)\right|^{2} d s \mathbb{E} \int_{0}^{b}\left\|B \bar{u}_{y}(s)\right\|^{2} d s \\
& +11 \int_{t_{1}}^{t_{2}}\left|\left(t_{2}-s\right)^{\alpha-1} \beta\left(t_{2}-s\right)\right|^{2} d s \mathbb{E} \int_{t_{1}}^{t_{2}}\left\|B \bar{u}_{y}(s)\right\|^{2} d s \\
& +11 \int_{0}^{t_{1}}\left|\left(t_{1}-s\right)^{\alpha-1}\left[\beta\left(t_{2}-s\right)-\beta\left(t_{1}-s\right)\right]\right|^{2} d s \mathbb{E} \int_{0}^{b}\left\|f\left(s, y_{s}+\widehat{\xi}_{s}\right)\right\|^{2} d s \\
& +11 \int_{0}^{t_{1}}\left|\left[\left(t_{2}-s\right)^{\alpha-1}-\left(t_{1}-s\right)^{\alpha-1}\right] \beta\left(t_{2}-s\right)\right|^{2} d s \mathbb{E} \int_{0}^{b}\left\|f\left(s, y_{s}+\widehat{\xi}_{s}\right)\right\|^{2} d s \\
& +11 \int_{t_{1}}^{t_{2}}\left|\left(t_{2}-s\right)^{\alpha-1} \beta\left(t_{2}-s\right)\right|^{2} d s \mathbb{E} \int_{t_{1}}^{t_{2}}\left\|f\left(s, y_{s}+\widehat{\xi}_{s}\right)\right\|^{2} d s \\
& +11 \mathbb{E} \int_{0}^{t_{1}}\left\|\left(t_{1}-s\right)^{\alpha-1}\left[\beta\left(t_{2}-s\right)-\beta\left(t_{1}-s\right)\right] g\left(s, y_{s}+\widehat{\xi}_{s}\right)\right\|^{2} d s \\
& +11 \mathbb{E} \int_{0}^{t_{1}}\left\|\left[\left(t_{2}-s\right)^{\alpha-1}-\left(t_{1}-s\right)^{\alpha-1}\right] \beta\left(t_{2}-s\right) g\left(s, y_{s}+\widehat{\xi}_{s}\right)\right\|^{2} d s \\
& +11 \mathbb{E} \int_{t_{1}}^{t_{2}}\left\|\left(t_{2}-s\right)^{\alpha-1} \beta\left(t_{2}-s\right) g\left(s, y_{s}+\widehat{\xi}_{s}\right)\right\|^{2} d s \\
& +11 \bar{s} \sum_{i=1}^{\bar{s}}\left\|\eta\left(t_{2}-t_{i}\right)-\eta\left(t_{1}-t_{i}\right)\right\|^{2} \mathbb{E}\left\|I_{i}\left(y\left(t_{i}^{-}\right)+\widehat{\xi}\left(t_{i}^{-}\right)\right)\right\|^{2} .
\end{aligned}
$$


The right hand of the inequality above tends to 0 as $t_{2} \rightarrow t_{1}$ by using Lebesgue dominated convergence theorem and (H1)-(H4) when $\|y\| \leqslant n_{0}$. If $t_{2} \rightarrow t_{1}$, then the right hand of the inequality above also tends to 0 . Therefore, the operator $G_{1}$ is equicontinuous on $J_{i}$.

Step 4. The conditions of Mönch hold. Assume that for any countable set $M \subseteq \mathrm{B}_{\mathrm{n}_{0}}, M \subseteq \overline{\operatorname{conv}}(\{0\} \cup$ $G_{1}(M)$ ), we show that the set $M$ is relatively compact. In fact, we only need to show that the Kuratowski measure of noncompactness of the set $M$ is 0 , that is, $\mu(M)=0$.

Suppose that $M=\left\{y^{(m)}\right\}_{m=1}^{\infty}, G_{1}(M)$ is equicontinuous on $J_{i}(i=1,2, \cdots, \bar{s})$ because $G\left(B_{n_{0}}\right)$ is equicontinuous on $\mathrm{J}_{\mathfrak{i}}(\mathfrak{i}=1,2, \cdots, \overline{\mathrm{s}})$.

By (H5), we have

$$
\begin{aligned}
& \mu_{\mathrm{u}}\left(\left\{\bar{u}_{y^{(m)}}(t)\right\}_{m=1}^{\infty}\right) \\
&=\mu\left(\left\{\Theta ^ { - 1 } \left[\bar{x}-\eta(b) \xi(0)-\lambda\left(b, y_{b}^{(m)}+\widehat{\xi}_{b}\right)-\int_{0}^{b}(b-s)^{\alpha-1} \beta(b-s) f\left(s, y_{s}^{(m)}+\widehat{\xi}_{s}\right) d s\right.\right.\right. \\
&-\int_{0}^{b}(b-s)^{\alpha-1} \beta(b-s) g\left(s, y_{s}^{(m)}+\widehat{\xi}_{s}\right) d W(s) \\
&\left.\left.\left.-\sum_{i=1}^{\bar{s}} \eta\left(b-t_{i}\right) I_{i}\left(y^{(m)}\left(t_{i}^{-}\right)+\widehat{\xi}\left(t_{i}^{-}\right)\right)\right](t)\right\}_{m=1}^{\infty}\right) \\
& \leqslant K_{\Theta}(t) K_{\lambda}(t) \mu\left(\left\{y^{(m)}\right\}_{m=1}^{\infty}\right) \\
&+K_{\Theta}(t) \mu\left(\left\{\int_{0}^{b}(b-s)^{\alpha-1} \beta(b-s) f\left(s, y_{s}^{(m)}+\widehat{\xi}_{s}\right) d s\right\}_{m=1}^{\infty}\right) \\
&+K_{\Theta}(t) \mu\left(\left\{\int_{0}^{b}(b-s)^{\alpha-1} \beta(b-s) g\left(s, y_{s}^{(m)}+\widehat{\xi}_{s}\right) d W(s)\right\}_{m=1}^{\infty}\right) \\
&+\sum_{i=1}^{\bar{s}} K_{\Theta}(t) L \mu\left(\left\{I_{i}\left(y^{(m)}\left(t_{i}^{-}\right)+\widehat{\xi}\left(t_{i}^{-}\right)\right)\right\}_{m=1}^{\infty}\right) .
\end{aligned}
$$

By Lemma 2.5 , Lemma 2.8 and (H1) (3), we obtain that

$$
\begin{aligned}
\mu\left(\left\{\int_{0}^{b}(b-s)^{\alpha-1} \beta(b-s) f\left(s, y_{s}^{(m)}+\widehat{\xi}_{s}\right) d s\right\}_{m=1}^{\infty}\right) \\
\quad \leqslant 2 \int_{0}^{b}(b-s)^{\alpha-1} \beta(b-s) \mu\left(\left\{f\left(s, y_{s}^{(m)}+\widehat{\xi}_{s}\right)\right\}_{m=1}^{\infty}\right) d s \\
\quad \leqslant 2 \int_{0}^{b}(b-s)^{\alpha-1} \beta(b-s) K_{f}(s) \sup _{-\tau \leqslant \theta \leqslant 0} \mu\left(\left\{y^{(m)}(s+\theta)+\widehat{\xi}(s+\theta)\right\}_{m=1}^{\infty}\right) d s \\
\quad \leqslant 2 \int_{0}^{b}(b-s)^{\alpha-1} \beta(b-s) K_{f}(s) \sup _{0 \leqslant \theta \leqslant s} \mu\left(\left\{y^{(m)}(s)+\widehat{\xi}(s)\right\}_{m=1}^{\infty}\right) d s \\
\leqslant 2 \int_{0}^{b}(b-s)^{\alpha-1} \beta(b-s) K_{f}(s) \sup _{0 \leqslant \theta \leqslant s} \mu\left(\left\{y^{(m)}(s)\right\}_{m=1}^{\infty}\right) d s \\
\leqslant \frac{2 L b^{\alpha}}{\Gamma(1+\alpha)}\left\|K_{f}\right\|_{L^{1}} \mu\left(\left\{y^{(m)}\right\}_{m=1}^{\infty}\right) .
\end{aligned}
$$

For any $y^{\prime}, y^{\prime \prime} \in B_{n_{0}}$, we have

$$
\begin{aligned}
\mathbb{E}\left\|\int_{0}^{b}(b-s)^{\alpha-1} \beta(b-s)\left[g\left(s, y_{s}^{\prime}+\widehat{\xi}_{s}\right)-g\left(s, y_{s}^{\prime \prime}+\widehat{\xi}_{s}\right)\right] d W(s)\right\|^{2} \\
\quad \leqslant \mathbb{E} \int_{0}^{b}(b-s)^{2 \alpha-2} \beta^{2}(b-s)\left\|g\left(s, y_{s}^{\prime}+\widehat{\xi}_{s}\right)-g\left(s, y_{s}^{\prime \prime}+\widehat{\xi}_{s}\right)\right\|^{2} d s \\
\leqslant \frac{\alpha^{2} L^{2} b^{2 \alpha-1}}{(2 \alpha-1) \Gamma(1+\alpha)^{2}} \sup _{0 \leqslant s \leqslant b} \mathbb{E}\left\|g\left(s, y_{s}^{\prime}+\widehat{\xi}_{s}\right)-g\left(s, y_{s}^{\prime \prime}+\widehat{\xi}_{s}\right)\right\|^{2} .
\end{aligned}
$$


By the properties of the Kuratowski measure of noncompactness of stochastic differential equations [6] and (H2) (3), we have

$$
\begin{array}{r}
\mu\left(\left\{\int_{0}^{b}(b-s)^{\alpha-1} \beta(b-s) g\left(s, y_{s}^{(m)}+\widehat{\xi}_{s}\right) d W(s)\right\}_{m=1}^{\infty}\right) \\
\leqslant \frac{\alpha L b^{\alpha-1 / 2}}{\sqrt{(2 \alpha-1)} \Gamma(1+\alpha)}\left\|K_{g}\right\|_{L^{1}} \mu\left(\left\{y^{(m)}\right\}_{m=1}^{\infty}\right)
\end{array}
$$

And by (H4) (2), we have

$$
\mu\left(\left\{I_{i}\left(y^{(m)}\left(t_{i}^{-}\right)+\widehat{\xi}\left(t_{i}^{-}\right)\right)\right\}_{m=1}^{\infty}\right)=\mu\left(\left\{I_{i}\left(y^{(m)}\left(t_{i}^{-}\right)\right)\right\}_{m=1}^{\infty}\right) \leqslant K_{i} \mu\left(\left\{y^{(m)}\right\}_{m=1}^{\infty}\right) .
$$

Substituting (3.6), (3.7), (3.8) into (3.5), we have by Lemma 2.9 and Lemma 2.10 that

$$
\begin{aligned}
& \mu_{\mathrm{u}}\left(\left\{\mathrm{u}_{\mathrm{y}^{(\mathfrak{m})}}\right\}_{\mathrm{m}=1}^{\infty}\right)=\max _{1 \leqslant i \leqslant \bar{s}} \sup _{\mathrm{t} \in \mathrm{J}_{\mathrm{i}}} \mu_{\mathrm{u}}\left(\left\{\mathrm{u}_{\mathrm{y}^{(\mathfrak{m})}}(\mathrm{t})\right\}_{\mathfrak{m}=1}^{\infty}\right) \\
& \leqslant\left\|\mathrm{K}_{\Theta}\right\|_{\mathrm{L}^{1}}\left(\frac{2 \mathrm{Lb}^{\alpha}}{\Gamma(1+\alpha)}\left\|\mathrm{K}_{\mathrm{f}}\right\|_{\mathrm{L}^{1}}+\frac{\alpha \mathrm{L} b^{\alpha-1 / 2}}{\sqrt{(2 \alpha-1)} \Gamma(1+\alpha)}\left\|\mathrm{K}_{\mathrm{g}}\right\|_{\mathrm{L}^{1}}\right. \\
& \left.+\sum_{i=1}^{\bar{s}} L K_{i}+\left\|K_{\lambda}\right\|_{L^{1}}\right) \mu\left(\left\{y^{(m)}\right\}_{m=1}^{\infty}\right) .
\end{aligned}
$$

Again by using the properties of the Kuratowski measure of noncompactness of stochastic differential equations [6], we have

$$
\begin{aligned}
\mu\left(\left\{G_{1} y^{(m)}(t)\right\}_{m=1}^{\infty}\right) \leqslant & \mu\left(\left\{\lambda\left(t, y_{t}^{(m)}+\widehat{\xi}_{t}\right)\right\}_{m=1}^{\infty}\right) \\
& +\mu\left(\left\{\int_{0}^{t}(t-s)^{\alpha-1} \beta(t-s) B \bar{u}_{y}(m)(s) d s\right\}_{m=1}^{\infty}\right) \\
& +\mu\left(\left\{\int_{0}^{t}(t-s)^{\alpha-1} \beta(t-s) f\left(s, y_{s}^{(m)}+\widehat{\xi}_{s}\right) d s\right\}_{m=1}^{\infty}\right) \\
& +\mu\left(\left\{\int_{0}^{t}(t-s)^{\alpha-1} \beta(t-s) g\left(s, y_{s}^{(m)}+\widehat{\xi}_{s}\right) d W(s)\right\}_{m=1}^{\infty}\right) \\
& +\mu\left(\left\{\sum_{i=1}^{\bar{s}} \eta\left(b-t_{i}\right) I_{i}\left(y^{(m)}\left(t_{i}^{-}\right)+\widehat{\xi}\left(t_{i}^{-}\right)\right)\right\}_{m=1}^{\infty}\right) .
\end{aligned}
$$

By Lemma 2.9, Lemma 2.10 and (3.9), we obtain that

$$
\begin{aligned}
\mu\left(\left\{G_{1} y^{(m)}\right\}_{m=1}^{\infty}\right)= & \max _{1 \leqslant i \leqslant s} \sup _{t \in J_{i}} \mu\left(\left\{G_{1} y^{(m)}(t)\right\}_{m=1}^{\infty}\right) \\
\leqslant & \left\|K_{\lambda}\right\|_{L^{1}} \mu\left(\left\{y^{(m)}\right\}_{m=1}^{\infty}\right) \\
& +\int_{0}^{b}(b-s)^{\alpha-1} \beta(b-s) K_{B} \mu_{U}\left(\left\{\bar{u}_{y^{(m)}}(s)\right\}_{m=1}^{\infty}\right) d s \\
& +\int_{0}^{b}(b-s)^{\alpha-1} \beta(b-s) K_{f}(s) \mu\left(\left\{f\left(s, y_{s}^{(m)}+\widehat{\xi}_{s}\right)\right\}_{m=1}^{\infty}\right) d s \\
& +\mu\left(\left\{\int_{0}^{b}(b-s)^{\alpha-1} \beta(b-s) g\left(s, y_{s}^{(m)}+\widehat{\xi}_{s}\right) d W(s)\right\}_{m=1}^{\infty}\right) \\
& +\sum_{i=1}^{\bar{s}} L_{K_{i} \mu}\left(\left\{y^{(m)}\right\}_{m=1}^{\infty}\right) \\
\leqslant & \left(1+\frac{L K_{B}\left\|K_{\Theta}\right\|_{L^{1}} b^{\alpha}}{\Gamma(1+\alpha)}\right) \times\left(\frac{2 L b^{\alpha}}{\Gamma(1+\alpha)}\left\|K_{f}\right\|_{L^{1}}+\sum_{i=1}^{\bar{s}} L K_{i}+\left\|K_{\lambda}\right\|_{L^{1}}\right. \\
& \left.+\frac{\alpha L b^{\alpha-1 / 2}}{\sqrt{(2 \alpha-1)} \Gamma(1+\alpha)}\left\|K_{g}\right\|_{L^{1}}\right) \mu\left(\left\{y^{(m)}\right\}_{m=1}^{\infty}\right) .
\end{aligned}
$$


Thus,

$$
\mu(M) \leqslant \mu\left(\overline{\operatorname{conv}}\left(\{0\} \cup G_{1}(M)\right)\right)=\mu\left(G_{1}(M)\right) \leqslant l \mu(M) .
$$

By (H6), we see that $\mu(M)=0$. So the operator $G_{1}$ has a fixed point $y$ in $B_{n_{0}}$. Then $x=y+\widehat{\xi}$ is the fixed point of the operator $F_{1}$. That is, the system (2.1) is controllable on J.

\section{Example}

As an application, we consider the following fractional impulsive neutral stochastic functional differential equation

$$
\left\{\begin{aligned}
&{ }^{c} D_{t}^{\alpha}(x(t, z)-\lambda(t, x(t-\tau, z)))= \frac{\partial}{\partial z} x(t, z)+m(z) u(t, z)+f(t, x(t-\tau, z)) \\
&+g(t, x(t-\tau, z)) \frac{d W(t)}{d t}, \quad t \in J:=[0, b], \\
&\left.\Delta x\right|_{t=t_{i}}=I_{i}\left(x\left(t_{i}^{-}\right)\right), \quad i=1,2, \cdots, \bar{s}, \\
& x_{0}=\xi(t) \in \mathcal{D}([-\tau, 0], H) .
\end{aligned}\right.
$$

Let $\mathrm{U}=\mathrm{H}=\mathrm{L}^{2}([0, \pi]), A: \mathrm{H} \rightarrow \mathrm{H}$ be defined by $A x=x^{\prime}$, whose domain

$$
\mathrm{D}(\mathrm{A})=\left\{x \in \mathrm{H}: \mathrm{x} \text { is absolutely continuous, and } x^{\prime} \in \mathrm{H}, \mathrm{x}(0)=0\right\} .
$$

A generates the semigroup $T(t)$. For any $x \in H, T(t) x(s)=x(t+s), T(t)$ is not a compact semigroup on $H$ (see [20]). For any bounded set $D \subset H, \mu(T(t) D) \leqslant \mu(D)$.

We consider the functions $\mathrm{f}, \lambda: \mathrm{J} \times \mathcal{D}([-\tau, 0] ; \mathrm{H}) \rightarrow \mathrm{H}$ and $\mathrm{g}: \mathrm{J} \times \mathcal{D}([-\tau, 0] ; \mathrm{H}) \rightarrow \mathcal{L}_{\mathrm{HS}}(\mathrm{H})$ defined by

$$
\begin{aligned}
& f(t, x(t-\tau))=\sin (x(t-\tau)) \\
& \lambda(t, x(t-\tau))=\cos (x(t-\tau)) \\
& g(t, x(t-\tau))=\frac{x(t-\tau)}{1+x(t-\tau)}
\end{aligned}
$$

and the impulsive function $\mathrm{I}_{\mathrm{i}}: \mathrm{H} \rightarrow \mathrm{H}$ and the control function $\mathrm{B}: \mathrm{H} \rightarrow \mathrm{H}$ defined by

$$
\begin{aligned}
& \mathrm{I}_{\mathfrak{i}}(x)=\cos (x), \\
& (\mathrm{Bu})(z)=\mathrm{m}(z) u(t, z), \quad z \in[0, \pi] .
\end{aligned}
$$

By these above functions, the assumptions (H1)-(H5) are satisfied. Moreover, we choose the appropriate parameters to make (H6) hold. Therefore, all the conditions in Theorem 3.1 have been satisfied. Thus, the system (4.1) is controllable on J.

\section{Acknowledgment}

The authors would like to thank the editor and the anonymous reviewers for their very useful comments and suggestions. The work is partially supported by the Natural Science Foundation of China under Grant 61374085 and the Fundamental Research Funds for the Central Universities, South-Central University for Nationalities under Grant CZW15113.

\section{References}

[1] R. P. Agarwal, M. Benchohra, S. Hamani, A survey on existence results for boundary value problems of nonlinear fractional differential equations and inclusions, Acta Appl. Math., 109 (2010), 973-1033. 1

[2] P. Balasubramaniam, J. P. Dauer, Controllability of semilinear stochastic delay evolution equations in Hilbert spaces, Int. J. Math. Math. Sci., 31 (2002), 157-166. 1 
[3] P. Balasubramaniam, J. Y. Park, P. Muthukumar, Approximate controllability of neutral stochastic functional differential systems with infinite delay, Stoch. Anal. Appl., 28 (2010), 389-400. 1

[4] H.-B. Bao, J.-D. Cao, Existence and uniqueness of solutions to neutral stochastic functional differential equations with infinite delay, Appl. Math. Comput., 215 (2009), 1732-1743. 1

[5] S. Das, D. Pandey, N. Sukavanam, Existence of solution and approximate controllability of a second-order neutral stochastic differential equation with state dependent delay, Acta Math. Sci. Ser. B Engl. Ed., 36 (2016), 1509-1523. 1

[6] A. Dehici, N. Redjel, Measure of noncompactness and application to stochastic differential equations, Adv. Difference Equ., 2016 (2016), 17 pages. 3, 3

[7] S. Duan, J.-H. Hu, Y. Li, Exact controllability of nonlinear stochastic impulsive evolution differential inclusions with infinite delay in Hilbert spaces, Int. J. Nonlinear Sci. Numer. Simul., 12 (2011), 23-33. 1

[8] T. Guendouzi, Existence and controllability of fractional-order impulsive stochastic system with infinite delay, Discuss. Math. Differ. Incl. Control Optim., 33 (2013), 65-87. 1

[9] D. J. Guo, Impulsive integral equations in Banach spaces and applications, J. Appl. Math. Stochastic Anal., 5 (1992), 111-122. 2.10

[10] H. P. Heinz, On the behaviour of measures of noncompactness with respect to differentiation and integration of vector-valued functions, Nonlinear Anal., 7 (1983), 1351-1371. 2.8

[11] L.-R. Huang, F.-Q. Deng, Razumikhin-type theorems on stability of neutral stochastic functional differential equations, IEEE Trans. Automat. Control, 53 (2008), 1718-1723 1

[12] Y.-G. Kao, Q.-X. Zhu, W.-H. Qi, Exponential stability and instability of impulsive stochastic functional differential equations with Markovian switching, Appl. Math. Comput., 271 (2015), 795-804. 1

[13] A. A. Kilbas, H. M. Srivastava, J. J. Trujillo, Theory and applications of fractional differential equations, North-Holland Mathematics Studies, Elsevier Science B.V., Amsterdam, (2006). 1

[14] V. Lakshmikantham, D. D. Ba inov, P. S. Simeonov, Theory of impulsive differential equations, Series in Modern Applied Mathematics, World Scientific Publishing Co., Inc., Teaneck, NJ, (1989). 1

[15] K.-X. Li, J.-G. Peng, Controllability of fractional neutral stochastic functional differential systems, Z. Angew. Math. Phys., 65 (2014), 941-959. 1

[16] X.-R. Mao, Razumikhin-type theorems on exponential stability of stochastic functional-differential equations, Stochastic Process. Appl., 65 (1996), 233-250. 1

[17] X.-R. Mao, Stochastic differential equations and their applications, Second edition, Woodhead Publishing Limited, Cambridge, (2007). 1

[18] K. S. Miller, B. Ross, An introduction to the fractional calculus and fractional differential equations, A Wiley-Interscience Publication, John Wiley \& Sons, Inc., New York, (1993). 1

[19] H. Mönch, Boundary value problems for nonlinear ordinary differential equations of second order in Banach spaces, Nonlinear Anal., 4 (1980), 985-999. 2.11

[20] A. Pazy, Semigroups of linear operators and applications to partial differential equations, Applied Mathematical Sciences, Springer-Verlag, New York, (1983). 4

[21] I. Podlubny, Fractional differential equations, An introduction to fractional derivatives, fractional differential equations, to methods of their solution and some of their applications, Mathematics in Science and Engineering, Academic Press, Inc., San Diego, CA, (1999). 1

[22] M. D. Quinn, N. Carmichael, An approach to nonlinear control problems using fixed-point methods, degree theory and pseudo-inverses, Numer. Funct. Anal. Optim., 7 (1984/85), 197-219. 3

[23] R. Sakthivel, R. Ganesh, S. Suganya, Approximate controllability of fractional neutral stochastic system with infinite delay, Rep. Math. Phys., 70 (2012), 291-311. 1

[24] R. Sakthivel, N. I. Mahmudov, J. J. Nieto, Controllability for a class of fractional-order neutral evolution control systems, Appl. Math. Comput., 218 (2012), 10334-10340. 1

[25] R. Sakthivel, S. Suganya, S. M. Anthoni, Approximate controllability of fractional stochastic evolution equations, Comput. Math. Appl., 63 (2012), 660-668. 1

[26] R. Triggiani, A note on the lack of exact controllability for mild solutions in Banach spaces, SIAM J. Control Optimization, 15 (1977), 407-411. 1, 3

[27] R. Triggiani, ddendum: "A note on the lack of exact controllability for mild solutions in Banach spaces" [SIAM J. Control Optim., 15 (1977), 407-411], SIAM J. Control Optim., 18 (1980), 98-99. 1

[28] Q. Wang, X.-Z. Liu, Impulsive stabilization of delay differential systems via the Lyapunov-Razumikhin method, Appl. Math. Lett., 20 (2007), 839-845. 1

[29] T. Yang, Impulsive systems and control: theory and applications, Nova Science Publishers, Inc., New York, (2001). 1

[30] R.-P. Ye, Existence of solutions for impulsive partial neutral functional differential equation with infinite delay, Nonlinear Anal., 73 (2010), 155-162. 2.9

[31] Y.-C. Zang, J.-P. Li, Approximate controllability of fractional impulsive neutral stochastic differential equations with nonlocal conditions, Bound. Value Probl., 2013 (2013), 13 pages. 1, 2.6

[32] Y. Zhou, F. Jiao, Existence of mild solutions for fractional neutral evolution equations, Comput. Math. Appl., 59 (2010), 1063-1077. 1, 2.5 